\title{
Emerging role of ONECUT2 in tumors (Review)
}

\author{
$\mathrm{JIA} \mathrm{YU}^{1 *}$, DONGYANG $\mathrm{LI}^{2 *}$ and HUA JIANG ${ }^{1}$ \\ Departments of ${ }^{1}$ Geriatrics and ${ }^{2}$ Urology, Shanghai East Hospital, \\ Tongji University School of Medicine, Shanghai 200120, P.R. China
}

Received March 10, 2020; Accepted August 21, 2020

DOI: $10.3892 / \mathrm{ol} .2020 .12192$

\begin{abstract}
One cut domain family member 2 (ONECUT2), also termed OC-2, is a newly discovered member of the ONECUT transcription factor family. As a transcription factor, ONECUT2 can widely regulate protein expression associated with cell proliferation, migration, adhesion, differentiation and cell material metabolism. Recent studies have revealed that ONECUT2 is associated with tumor cell proliferation, angiogenesis and metastasis; it is also associated with epithelial-mesenchymal transition in cancer cells. The present review examines the distribution and expression of ONECUT2 in a variety of tumors, its oncogenic role in tumor progression and the possible mechanisms of regulation. Given the emerging role of ONECUT2 in the development and progression of tumors, ONECUT2 might be a promising target for tumor therapy.
\end{abstract}

\section{Contents}

1. Introduction

2. Emerging role of ONECUT2 in tumors

3. ONECUT2 drives prostate cancer progression to castration-resistant prostate cancer (CRPC)

4. ONECUT2 affects colorectal cancer (CRC) progression by regulating epithelial-mesenchymal transition (EMT)

5. ONECUT2 may be involved in the inhibitory pathways of hepatocellular carcinoma (HCC)

6. ONECUT2 promotes ovarian cancer cell proliferation and migration

7. ONECUT2 induces stem cell properties after chemotherapy in breast cancer

Correspondence to: Professor Hua Jiang, Department of Geriatrics, Shanghai East Hospital, Tongji University School of Medicine, 150 Jimo Road, Pudong New Area, Shanghai 200120, P.R. China

E-mail: huajiang2013@tongji.org.cn

${ }^{*}$ Contributed equally

Key words: ONECUT2, proliferation, metastasis, transdifferentiation, cancer stemness
8. ONECUT2 promotes RAS-driven lung cancer and promotes stem cell properties

9. ONECUT2 is a promising predictor of bladder cancer

10. Future prospects

11. Conclusions

\section{Introduction}

In 1999, Jacquemin et al (1) first reported the identification and characterization of human one cut domain family member 2 (ONECUT2), also known as OC-2. The other two members of the ONECUT transcription factor family are hepatocyte nuclear factor 6 and ONECUT3 (1). The ONECUT2 gene is located on chromosome 18 of the human genome, consisting of two exons and one intron (Fig. 1) (1). ONECUT2 protein is characterized by one cut domain and an atypical homologous domain; the protein binds to specific DNA sequences to stimulate the expression of target genes (1). ONECUT2 is mainly distributed in the gallbladder, duodenum, liver and small intestines in human tissues, and has low expression levels in the brain, pancreas, stomach and testes (1).

As a transcription factor, ONECUT2 can widely regulate the protein expression associated with cell proliferation, migration, adhesion and differentiation, thus being involved in the regulation of the development of an organism (2-9).

\section{Emerging role of ONECUT2 in tumors}

The role of ONECUT2 in tumorigenesis has been investigated. Table I summarizes the expression levels of ONECUT2 in different types of tumor, the effect of ONECUT2 on tumor development and the potential mechanisms of its regulation. The levels of ONECUT2 protein are elevated in prostate cancer $(10,11)$, colorectal cancer $(12,13)$, hepatocellular carcinoma (14), ovarian cancer (15) and lung cancer (16). Increased ONECUT2 expression is associated with regulation of tumor cell proliferation (17), metastasis (15) and differentiation (18). However, the levels of ONECUT2 are decreased in breast cancer and this is associated with inducing tumor stemness (19). Additionally, increased levels of ONECUT2 gene methylation in urine may be a promising predictor of bladder cancer (20-22). 
3. ONECUT2 drives prostate cancer progression to castration-resistant prostate cancer (CRPC)

Prostate cancer ( $\mathrm{PCa})$ is the second most commonly diagnosed cancer, with an incidence rate of $13.5 \%$, and the fifth most lethal cause of cancer-associated death, with a mortality rate of $6.7 \%$ among men worldwide, as reported in 2018 (23). Studies have shown increased expression of ONECUT2 in prostate cancer $(10,11)$. Immunocoprecipitation experiements show that ONECUT2 and androgen receptor (AR) coexist, indicating that they may interact with each other (24). Dihydrotestosterone stimulation can increase the binding of ONECUT2 and AR to rs7463708, a single nucleotide polymorphism associated with prostate cancer risk (25). rs7463708 then interacts with the Prostate Cancer Associated Transcript 1 (PCAT1) promoter and upregulates the expression of PCAT1, which is an androgen late-response gene (24). PCAT1 combines with AR and lysine-specific demethylase 1 in an androgen-dependent manner (24). Through interaction with chromatin, PCAT1 upregulates the expression of GNMT and DHCR24, thus promoting tumor cell proliferation and tumor growth (24). This process may be a way to promote the occurrence of prostate cancer (Fig. 2).

Androgen deprivation therapy is the core therapy for advanced and metastatic prostate cancer, but through a variety of mechanisms, it eventually develops into resistance to androgen therapy, namely CRPC (26,27). Nearly one-fifth of metastatic CRPC (mCRPC) cases exhibit small cell neuroendocrine (NE) pathological characteristics after treatment with effective AR pathway inhibitors (28). One of the mechanisms of resistance is the differentiation into NE prostate cancer (NEPC). NEPC is a lethal invasive subtype of prostate cancer, characterized by the loss of AR signal during NE trans-differentiation (29).

The expression of the AR gene is negatively correlated with the cell proliferation signal in $\mathrm{mCRPC}$, and the decrease in AR activity is consistent with disease progression (30), which strongly implies that an alternative transcription pathway is in action. Rotinen et al (18) hypothesized that ONECUT2 is a targetable master regulator of CRPC. Computational modeling was applied to 38 transcriptome datasets from 2,115 PC cases, including 260 samples of mCRPC. The modeling predicted that ONECUT2 activity is comparable to that of histone-lysine N-methyltransferase EZH2 (EZH2), a known CRPC driver, and that ONECUT2 forms a network with other key transcription factors, including POU domain class 5 transcription factor 1, paired box protein Pax-5, AR and EZH2. In mCRPC, the expression levels and activity of ONECUT2 are negatively correlated with those of AR, and chromatin immunoprecipitation sequencing revealed ONECUT2 binding to the AR promoter; therefore, ONECUT2 may act as a direct repressor of AR gene expression (18). After ONECUT2 expression is enhanced, the expression of AR and the AR regulatory genes KLK3, KLK2 and EHF is downregulated, and the expression of the prostate-specific antigen (PSA) gene and the PSA enhancer activity of the classical regulatory target of AR are also inhibited (18). ONECUT2 can activate the transcription program of mCRPC, and endogenous ONECUT2 can combine with the forkhead box A1 (FOXA1) promoter and gene to inhibit the expression of FOXA1 mRNA and protein.
FOXA1, an inhibitor of NE differentiation, disappears with the development of mCRPC (31). These results indicate that ONECUT2 suppresses the AR axis to promote the emergence of NEPC.

RE1-silencing transcription factor (REST) is a master repressor of neuronal differentiation (32) and REST loss is implicated in NE-CRPC (29). Chromatin immunoprecipitation-quantitative PCR demonstrates the direct binding of REST at the ONECUT2 promoter, and there is an inverse association between ONECUT2 and REST expression in NEPC, suggesting that REST is a disease-related expression inhibitor of ONECUT2 (29). Paternally expressed gene 10 (PEG10) has been identified as a driving factor for the transition from adenocarcinoma (ADC) to NEPC in the LTL331 model, a patient-derived xenograft model of NEPC trans-differentiation (33). During the transition, ONECUT2 combines with the PEG10 promoter and increases the PEG10 mRNA level, suggesting that ONECUT2 is the direct upstream activator of PEG10 transcription. It can be concluded that ONECUT2 is located between REST and PEG10 during the transformation of prostate ADC into NEPC (18).

Using structure-based drug design, Rotinen et al (18) constructed a three-dimensional model of the ONECUT2-HOX domain and screened out CXRM617 as a small molecular inhibitor of ONECUT2. In vitro, CXRM617 can inhibit the proliferation and induce the apoptosis of prostate cancer cell lines with high expression of ONECUT2. CXRM617 significantly reduces the tumor volume and weight, and reduces the occurrence and growth of diffuse metastasis in vivo. CXRM617 can be used as the main candidate drug for the development of mCRPC therapy (34).

Guo et al (24) proposed that ONECUT2 drives tumor aggressiveness partially through synergizing with hypoxia. Specifically, ONECUT2 activates SMAD3, which regulates the hypoxia signal by regulating HIF1 $\alpha$ chromatin binding, resulting in a higher degree of hypoxia in NEPC than in prostate ADC. The use of TH-302, a hypoxia-activated prodrug, can significantly reduce the growth of NEPC.

Previous research notes that ONECUT2 is a novel target for the treatment of CRPC $(18,29)$ (Fig. 3). Further preclinical studies are required to determine the prostate cancer types most likely to respond to ONECUT2 inhibition, as well as to understand ONECUT2-driven castration resistance mechanisms, identify predictive biomarkers, and understand the role of ONECUT2 in tumor heterogeneity and tumor evolution.

\section{ONECUT2 affects colorectal cancer (CRC) progression by regulating epithelial-mesenchymal transition (EMT)}

CRC is one of the most common gastrointestinal malignancies, with a 5-year survival rate of $\sim 55 \%$. Upregulated ONECUT2 expression in CRC $(12,13)$ is associated with lymph node metastasis, and a low level of ONECUT2 is associated with a good prognosis (13), but the specific mechanism involved has not been clarified.

EMT is one of the well-defined processes involved during the invasion and distant metastasis of primary epithelial tumors (35). MicroRNAs (miRNAs/miRs) of the miR-200 family have been experimentally shown to regulate EMT $(12,36)$. miR-429, as a member of the miR-200 
Table I. Level and effect of ONECUT2 in different tumors.

\begin{tabular}{|c|c|c|c|c|c|c|}
\hline Cancer type & ONECUT2 level & Effect of ONECUT2 & Upstream & $\begin{array}{l}\text { Downstream } \\
\text { targets }\end{array}$ & Pathway & Inhibitor \\
\hline Prostate cancer & $\uparrow$ & $\begin{array}{l}\text { Promotes proliferation; regulates } \\
\text { neuroendocrine differentiation }\end{array}$ & REST & $\begin{array}{l}\text { AR, FOXA1, } \\
\text { PEG10 }\end{array}$ & $\begin{array}{l}\text { SMAD3 } \\
\text { pathway }\end{array}$ & $\begin{array}{l}\text { CSRM617, } \\
\text { TH302 }\end{array}$ \\
\hline Colorectal cancer & $\uparrow$ & $\begin{array}{l}\text { Promotes proliferation, EMT } \\
\text { and lymph node metastasis }\end{array}$ & $\operatorname{miR}-429$ & & & \\
\hline $\begin{array}{l}\text { Hepatocellular } \\
\text { carcinoma }\end{array}$ & $\uparrow$ & Predicts poor survival time & & & & \\
\hline Ovarian cancer & $\uparrow$ & Promotes proliferation and EMT & & VEGF & $\begin{array}{l}\text { AKT/ERK } \\
\text { pathway }\end{array}$ & \\
\hline Lung cancer & $\uparrow$ & Promotes tumor stemness & miR-543 & & & \\
\hline Breast cancer & $\downarrow$ & Induces tumor stemness & $\begin{array}{l}\text { miR-9-5p, } \\
\text { miR-195-5p, } \\
\text { miR-203a-3p }\end{array}$ & & $\begin{array}{l}\text { EV-miRNA } \\
\text { ONECUT2 axis }\end{array}$ & \\
\hline Bladder cancer & $\begin{array}{c}\uparrow \text { (methylation } \\
\text { level) }\end{array}$ & Predicts and diagnoses disease & & & & \\
\hline
\end{tabular}

ONECUT2, one cut domain family member 2; REST, RE1-silencing transcription factor; AR, androgen receptor; FOXA1, forkhead box A1; PEG10, paternally expressed gene 10; EMT, epithelial-mesenchymal transition; miR, microRNA; EV, extracellular vesicle.

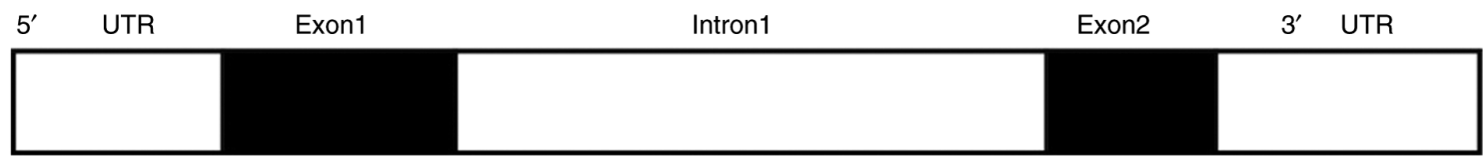

Figure 1. Schematic view of the One cut domain family member 2 gene. UTR, untranslated region.

family, can inhibit the tumorigenicity of CRC cells in vivo; this miRNA can reverse the change in EMT induced by TGF- $\beta$ and inhibit EMT inducible factors (such as ZEB1, ZEB2, SNAI and SLUG) (12). Sun et al (12) confirmed that ONECUT2 is the target gene of miR-429. High expression of ONECUT2 can reverse the inhibition of EMT inducible factors and the inhibition of migration and invasion mediated by miR-429. Ranković et al (13) also showed that downregulation of the miR-200 family is observed at the invasive front rather than in the central region of tumors in $\mathrm{CRC}$, and that the expression of ONECUT2 was correlated with nodal metastases (13). These results suggest that the miR-200 family and their target gene ONECUT2 contribute to the transition from adenoma to CRC and to the development of metastases.

Hui et al (37) constructed IncRNA-miRNA-mRNA expression networks using the Gene Expression Omnibus database and found that ONECUT2 associates with multiple miRNAs in the competing endogenous RNA network, including has-miR139-5p in CRC. Has-miR139-5p expression levels are significantly decreased within colon tumors and are correlated with tumor grade. Has-miR-139-5p, by directly targeting the BCL2 pathway, can reduce tumor metastasis and drug sensitivity in CRC. Has-miR-139-5p interacts with ONECUT2 and the expression levels are negatively correlated, suggesting that has-miR-139-5p and ONETCUT2 may have a similar regulatory mechanism to that between has-miR-139-5p and BCL2.

\section{ONECUT2 may be involved in the inhibitory pathways of hepatocellular carcinoma (HCC)}

The prognosis of HCC remains poor due to tumor progression and a high tumor recurrence rate (38). miR-9 dysregulation is implicated in a number of human malignancies. miR-9 inhibits ovarian cancer cell growth (39), regulates gastric cancer cell growth and exhibits tumor suppressive activity in human gastric cancer pathogenesis (40), promotes tumor metastasis in esophageal squamous cell carcinoma (41) and increases cervical cancer cell motility (42). A previous in vitro study revealed that miR-9 restoration retards HCC cell proliferation and migration (14). Zhang et al (14) confirmed that ONECUT2, IGF2BP1 and ANXA2 are miR-9 targets, and that they are aberrantly upregulated in HCC. The upregulation of ONECUT2, IGF2BP1 and IL-6 is significantly correlated with poor post-surgery survival rate. It is suggested that miR-9 acts as a tumor suppressor by inhibiting a series of target genes, including those of the miR-9/IGF2BP1/AKT/ERK axis.

\section{ONECUT2 promotes ovarian cancer cell proliferation and migration}

Ovarian cancer is one of the deadliest of all the gynecological malignancies. ONECUT2 expression is significantly increased in malignant ovarian ADC cell lines and in most malignant ovarian cancer tissues; however, it is significantly decreased in less malignant cancer cell lines, suggesting that the abnormal 


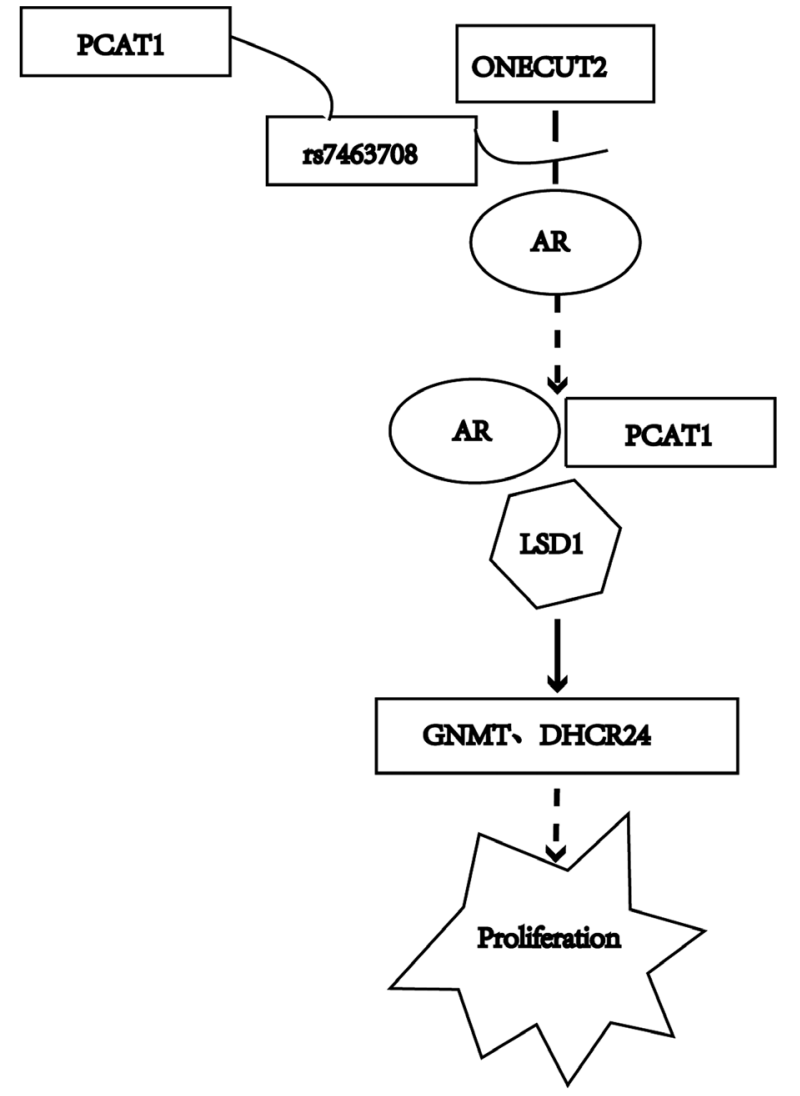

Figure 2. Pathways of ONECUT2 promoting prostate cancer cell proliferation. AR, androgen receptor. ONECUT2 binds with AR to rs7463708, which then interacts with PCAT1 promoter and upregulates PCAT1 expression; PCAT1 combines with AR and LSD1, and then PCAT1 upregulates GNMT and DHCR24 expression, thus promoting tumor cell proliferation. Different shapes indicate different proteins or molecules. Continuous and curved lines indicate direct interaction, while dashed lines indicate indirect interaction. ONECUT2, One cut domain family member 2; GNMT, glycine N-methyltransferase; PCAT1, Prostate Cancer Associated Transcript 1; AR, androgen receptor; LSD1, lysine-specific demethylase 1.

expression of ONECUT2 is closely associated with the degree of deterioration.

Silencing ONECUT2 expression using siRNA was shown to significantly decrease the tumor inhibition rate by $\sim 73 \%$ in an ovarian cancer xenograft mouse model (15). Silencing ONECUT2 could inhibit the phosphorylation of AKT and ERK, implying that ONECUT2 may promote the proliferation of ovarian cancer cells through the AKT/ERK signaling pathway (17).

After silencing ONECUT2 expression, the migration rate and invasion rate of cancer cells decreases significantly. ONECUT2 silencing upregulates the E-cadherin level and downregulates the $\mathrm{N}$-cadherin level, and the change in EMT-related markers is consistent with the characteristics of EMT. ONECUT2 silencing also significantly inhibits the expression of VEGF. These results suggest that ONECUT2 may promote tumor migration and invasion by activating EMT and promoting angiogenesis (15).

\section{ONECUT2 induces stem cell properties after chemotherapy in breast cancer}

Cancer stem cells (CSCs) have been proposed as the driving force of tumorigenesis and as the seeds of metastases (43), and

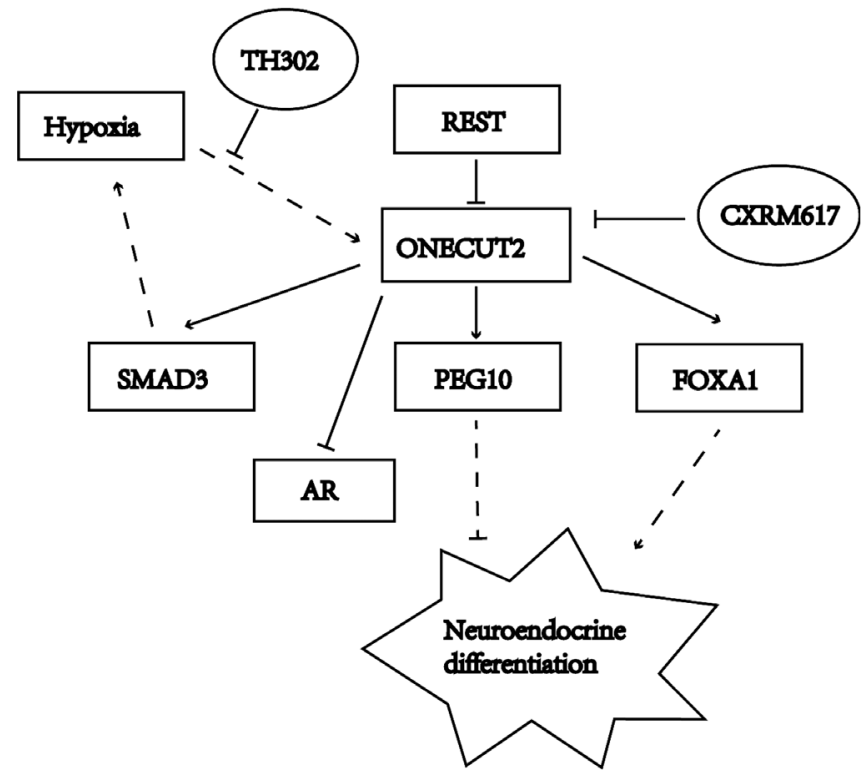

Figure 3. Pathways of ONECUT2 driving neuroendocrine differentiation. ONECUT2 suppresses the AR axis and inhibits FOXA1 expression, an inhibitor of neuroendocrine differentiation, to promote the emergence of NEPC. ONECUT2 is located between REST and PEG10 during the transformation of prostate adenocarcinoma into NEPC. ONECUT2 regulates hypoxia signaling by activating SMAD3 to drive NEPC, and the use of TH-302, a hypoxia-activated prodrug, can significantly reduce the growth of NEPC. Different shapes indicate different proteins or molecules. Continuous lines indicate direct interaction, while dashed lines indicate indirect interaction. REST, RE1-silencing transcription factor; AR, androgen receptor; ONECUT2, one cut domain family member 2 ; PEG10, paternally expressed gene 10; NEPC, neuroendocrine prostate cancer; FOXA1, forkhead box A1.

they tend to be resistant to chemoradiotherapy and molecular targeted therapy. ONECUT2 may be involved in inducing the stemness of tumor cells.

Chemotherapy induces breast cancer cells to secrete a variety of miRNAs, including miR-9-5p, miR-195-5p and miR-203a-3p. These miRNAs are wrapped in extracellular vesicles (EVs), which enable cancer cells to communicate with each other and with non-cancerous cells in tumor pathogenesis and in response to therapies, resulting in resistance to treatment. The aforementioned three miRNAs target ONECUT2 at the same time and downregulate its expression, leading to induction of CSC traits and upregulation of the expression of stem cell characteristic-related genes. The results suggest a possible mechanism of cancer cells communicating with each other and adapting themselves to survive in response to cytotoxic therapy. Inhibition of these miRNAs or recovery of ONECUT2 expression can eliminate the CSC stimulation of EVs by cancer cells after chemotherapy. This suggests a potential strategy, namely, blocking the EV-miRNA ONECUT2 axis to maximize the anticancer effect of chemotherapy and reduce chemoresistance in cancer management (19).

\section{ONECUT2 promotes RAS-driven lung cancer and promotes stem cell properties}

Lung cancer encompasses a heterogenous group of malignancies. ONECUT2 expression across various subtypes of lung cancer was analyzed using the publicly available dataset GSE30219 (16). According to this study, ONECUT2 is aberrantly activated in lung cancer, including small cell lung 
cancer (SCLC), large cell NE lung cancer, lung carcinoid tumor and lung ADC (16). The upregulation of ONECUT2 is significantly associated with shorter overall survival times in 60 patients with RAS-driven ADC and 115 patients with RAS-activated ADC (16). Overexpression of ONECUT2 can promote the malignant growth, invasion and adhesion of A549 lung cancer cells in vitro (16). A549 is a human lung ADC cell line with a homozygous KRAS mutation. In vivo, ONECUT2 overexpression can enhance xenograft tumorigenesis and bone metastasis (16). Further integrative transcriptomics and epigenomics analyses indicate that ONECUT2 promotes the transdifferentiation of lung cancer cells by preferentially targeting and regulating the activity of bivalent chromatin domains through modulation of polycomb repressive complex 2 occupancy (16). The specific mechanism behind the oncogenic role of ONECUT2 in the RAS-driven progression and metastasis of lung cancer should be further investigated, which may provide potential therapies for RAS-driven lung cancer.

lncRNA plays an important role in the occurrence and development of tumors due to its transcription, epigenetic modification or chromosomal abnormalities (44). The lncRNA THUMPD3 antisense RNA1 (THUMPD3-AS1) has oncogene activity and is aberrantly upregulated in non-SCLC (NSCLC) tissues, correlating with TNM stage and recurrence. THUMPD3-AS1 promotes NSCLC proliferation and self-renewal capacity by acting as an endogenous sponge of miR-543, and ONECUT2 is a direct downstream target of miR-543, which suggests that ONECUT2 can promote stem cell properties in NSCLC (45).

\section{ONECUT2 is a promising predictor of bladder cancer}

Hematuria is the most common finding in bladder cancer (BCa), and it is estimated that approximately $3-15 \%$ of the patients with hematuria have urinary tract cancer (with the most common being BCa) (46). Cystoscopy is considered the gold standard for the detection of $\mathrm{BCa}$ in patients with asymptomatic hematuria (47), however it is expensive and invasive in nature. Several studies have aimed to establish urine testing methods to screen patients with asymptomatic hematuria for the detection of bladder cancer. DNA hypermethylation is a common epigenetic regulation in malignancies. Beukers et al (20) stratified 167 patients by age and analyzed the methylation of five genes, ONECUT2 included. The results showed that ONECUT2 methylation is present in patients with urothelial carcinoma and that the methylation rate is higher in elderly individuals than in the young. van Kessel et al (21) included 200 patients from three European countries and extracted DNA from urine for methylation analysis. Combining the methylation markers (OTX1, ONECUT2 and TWIST1) and mutation markers (FGFR3, TERT and HRAS) with age contributed to a validated accurate prediction model, which was suggested to reduce the requirement for diagnostic cystoscopy by $77 \%$. Wu et al (22) collected and evaluated 192 urine samples from patients with hematuria in China. A combination methylation assay of four genes (HOXA9, PCDH17, POU4F2 and ONECUT2) was developed and indicated that $\sim 60 \%$ of cystoscopies are unnecessary. ONECUT2 is a promising predictor of bladder cancer. However, thus far, studies on the mechanism of ONECUT2 in the development of bladder cancer are lacking, and further investigation is needed.

\section{Future prospects}

Increasing evidence demonstrates that the expression of ONECUT2 affects the development and progression of malignant tumors, and that expression level may display great potential in the diagnosis, prognosis and treatment of tumors. Research into the regulatory mechanics of the ONECUT2 gene has enormous potential. ONECUT2 has been identified as a driver of NEPC. However, further validation in genetically engineered mouse models or in pre-clinical $\mathrm{PCa}$ organoid models is warranted. Establishing which subtype of prostate cancer responds to ONECUT2 inhibitors in clinical treatment will be critical for the majority of CRPC patients. CSCs play a potential key role in the progression, metastasis and chemoresistance of tumors. ONECUT2 may be involved in inducing the stemness of tumor cells. Regulation of ONECUT2 via miRNA targeting and other mechanisms would reprogram the levels of gene expression towards a pluripotent state. Future studies deciphering the spatiotemporal pattern of ONECUT2 expression and its regulation during tumor progression may provide novel insights into improved cancer management. ONECUT2 represents a good candidate as a reliable biomarker for diagnosis in bladder cancer. However, at present, it can only be used in combination with other biomarkers for bladder cancer diagnosis, and the clinical application of ONECUT2 still requires further investigation and optimization.

\section{Conclusions}

ONECUT2 is a transcription factor associated with development, and recent studies have shown that the aberrant expression of ONECUT2 in cancer is relevant with cancer progression. ONECUT2 has gained attention from research into prostate cancer and it interacts with AR to promote prostate cancer cell proliferation. Meanwhile, in CRPC, ONECUT2 inhibits the AR axis and drives NE trans-differentiation. This dual role of ONECUT2 could help explain how certain prostate cancer types evade hormone therapy and become more aggressive, and indicates that ONECUT2 is a new target for the treatment of CRPC. ONECUT2 also plays a role in the progression of other tumors by regulating cell proliferation, metastasis and EMT. CSCs are associated with tumor metastasis, and ONECUT2 may be involved in inducing the characteristics of CSCs during chemoradiotherapy resistance. Blocking the effect of ONECUT2 may provide a new method for the treatment of cancer and the elimination of chemotherapy resistance.

\section{Acknowledgements}

Not applicable.

\section{Funding}

This study was supported by the Fundamental Research Funds for the Central Universities (grant no. 22120180603) and the Important Weak Subject Construction Project of Pudong Health and Family Planning Commission of Shanghai (grant no. PWZbr2017-06). 


\section{Availability of data and materials}

Not applicable.

\section{Authors' contributions}

JY and DL searched the literature and wrote the manuscript. HJ revised the manuscript. All authors read and approved the final manuscript.

\section{Ethics approval and consent to participate}

Not applicable.

\section{Patient consent for publication}

Not applicable.

\section{Competing interests}

The authors declare that they have no competing interests.

\section{References}

1. Jacquemin P, Lannoy VJ, Rousseau GG and Lemaigre FP: OC-2, a novel mammalian member of the ONECUT class of homeodomain transcription factors whose function in liver partially overlaps with that of hepatocyte nuclear factor-6. J Biol Chem 274: 2665-2671, 1999.

2. Dusing MR, Maier EA, Aronow BJ and Wiginton DA: Onecut-2 knockout mice fail to thrive during early postnatal period and have altered patterns of gene expression in small intestine. Physiol Genomics 42: 115-125, 2010.

3. Maier EA, Dusing MR and Wiginton DA: Temporal regulation of enhancer function in intestinal epithelium: A role for Onecut factors. J Biol Chem 281: 32263-32271, 2006.

4. Margagliotti S, Clotman F, Pierreux CE, Beaudry JB, Jacquemin P, Rousseau GG and Lemaigre FP: The Onecut transcription factors HNF-6/OC-1 and OC-2 regulate early liver expansion by controlling hepatoblast migration. Dev Biol 311: 579-589, 2007.

5. Clotman F, Jacquemin P, Plumb-Rudewiez N, Pierreux CE, Van der Smissen P, Dietz HC, Courtoy PJ, Rousseau GG and Lemaigre FP: Control of liver cell fate decision by a gradient of TGF beta signaling modulated by Onecut transcription factors. Genes Dev 19: 1849-1854, 2005.

6. Laudadio I, Manfroid I, Achouri Y, Schmidt D, Wilson MD, Cordi S, Thorrez L, Knoops L, Jacquemin P, Schuit F, et al: A feedback loop between the liver-enriched transcription factor network and miR-122 controls hepatocyte differentiation. Gastroenterology 142: 119-129, 2012.

7. Goetz JJ, Martin GM, Chowdhury R and Trimarchi JM: Onecut1 and Onecut2 play critical roles in the development of the mouse retina. PLoS One 9: e110194, 2014.

8. Sapkota D, Chintala H, Wu F, Fliesler SJ, Hu Z and Mu X: Onecut1 and Onecut 2 redundantly regulate early retinal cell fates during development. Proc Natl Acad Sci USA 111: E4086-E4095, 2014

9. van der Raadt J, van Gestel SHC, Nadif Kasri N and Albers CA: ONECUT transcription factors induce neuronal characteristics and remodel chromatin accessibility. Nucleic Acids Res 47: 5587-5602, 2019.

10. Yamamoto $\mathrm{F}$ and Yamamoto M: Scanning copy number and gene expression on the 18q21-qter chromosomal region by the systematic multiplex PCR and reverse transcription-PCR methods. Electrophoresis 28: 1882-1895, 2007.

11. Leyten GH, Hessels D, Smit FP, Jannink SA, de Jong H, Melchers WJ, Cornel EB, de Reijke TM, Vergunst H, Kil P, et al: Identification of a candidate gene panel for the early diagnosis of prostate cancer. Clin Cancer Res 21: 3061-3070, 2015.

12. Sun Y, Shen S, Liu X, Tang H, Wang Z, Yu Z, Li X and Wu M: MiR-429 inhibits cells growth and invasion and regulates EMT-related marker genes by targeting Onecut 2 in colorectal carcinoma. Mol Cell Biochem 390: 19-30, 2014.
13. Ranković B, Zidar N, Zlajpah M and Bostjancic E: Epithelial-mesenchymal transition-related MicroRNAs and their target genes in colorectal cancerogenesis. J Clin Med 8: 1603 , 2019.

14. Zhang J, Cheng J, Zeng Z, Wang Y, Li X, Xie Q, Jia J, Yan Y, Guo Z, Gao J, et al: Comprehensive profiling of novel microRNA-9 targets and a tumor suppressor role of microRNA-9 via targeting IGF2BP1 in hepatocellular carcinoma. Oncotarget 6: 42040-42052, 2015.

15. Lu T, Wu B, Yu Y, Zhu W, Zhang S, Zhang Y, Guo J and Deng N: Blockade of ONECUT2 expression in ovarian cancer inhibited tumor cell proliferation, migration, invasion and angiogenesis. Cancer Sci 109: 2221-2234, 2018.

16. Ma Q, Wu K, Li H, Li H, Zhu Y, Hu G, Hu L and Kong X: ONECUT2 overexpression promotes RAS-driven lung adenocarcinoma progression. Sci Rep 9: 20021, 2019.

17. Abid MR, Guo S, Minami T, Spokes KC, Ueki K, Skurk C, Walsh K and Aird WC: Vascular endothelial growth factor activates PI3K/Akt/forkhead signaling in endothelial cells. Arterioscler Thromb Vasc Biol 24: 294-300, 2004.

18. Rotinen M, You S, Yang J, Coetzee SG, Reis-Sobreiro M, Huang WC, Huang F, Pan X, Yáñez A, Hazelett DJ, et al: ONECUT2 is a targetable master regulator of lethal prostate cancer that suppresses the androgen axis. Nat Med 24: 1887-1898, 2018.

19. Shen M, Dong C, Ruan X, Yan W, Cao M, Pizzo D, Wu X, Yang L, Liu L, Ren X and Wang SE: Chemotherapy-Induced Extracellular Vesicle miRNAs promote breast cancer stemness by targeting ONECUT2. Cancer Res 79: 3608-3621, 2019.

20. Beukers W, Hercegovac A, Vermeij M, Kandimalla R, Blok AC, van der Aa MM, Zwarthoff EC and Zuiverloon TC: Hypermethylation of the polycomb group target gene PCDH7 in bladder tumors from patients of all ages. J Urol 190: 311-316, 2013.

21. van Kessel KE, Beukers W, Lurkin I, Ziel-van der Made A, van der Keur KA, Boormans JL, Dyrskjot L, Marquez M, Ørntoft TF, Real FX, et al: Validation of a DNA methylation-mutation urine assay to select patients with hematuria for cystoscopy. J Urol 197: 590-595, 2017.

22. Wu Y, Jiang G, Zhang N, Liu S, Lin X, Perschon C, Zheng SL, Ding Q, Wang X, Na R, et al: HOXA9, PCDH17, POU4F2, and ONECUT2 as a urinary biomarker combination for the detection of bladder cancer in chinese patients with hematuria. Eur Urol Focus 6: 284-291, 2018.

23. Bray F, Ferlay J, Soerjomataram I, Siegel RL, Torre LA and Jemal A: Global cancer statistics 2018: GLOBOCAN estimates of incidence and mortality worldwide for 36 cancers in 185 countries. CA Cancer J Clin 68: 394-424, 2018.

24. Guo H, Ci X, Ahmed M, Hua JT, Soares F, Lin D, Puca L, Vosoughi A, Xue H, Li E, et al: ONECUT2 is a driver of neuroendocrine prostate cancer. Nat Commun 10: 278, 2019.

25. Chung S, Nakagawa H,Uemura M,Piao L, Ashikawa K, Hosono N, Takata R, Akamatsu S, Kawaguchi T, Morizono T, et al: Association of a novel long non-coding RNA in 8q24 with prostate cancer susceptibility. Cancer Sci 102: 245-252, 2011.

26. Pienta KJ and Bradley D: Mechanisms underlying the development of androgen-independent prostate cancer. Clin Cancer Res 12: 1665-1671, 2006.

27. Harris WP, Mostaghel EA, Nelson PS and Montgomery B: Androgen deprivation therapy: Progress in understanding mechanisms of resistance and optimizing androgen depletion. Nat Clin Pract Urol 6: 76-85, 2009.

28. Aggarwal R, Huang J, Alumkal JJ, Zhang L, Feng FY, Thomas GV, Weinstein AS, Friedl V, Zhang C, Witte ON, et al: Clinical and genomic characterization of treatment-emergent small-cell neuroendocrine prostate cancer: A Multi-institutional prospective study. J Clin Oncol 36: 2492-2503, 2018.

29. Lapuk AV, Wu C, Wyatt AW, McPherson A, McConeghy BJ, Brahmbhatt S, Mo F, Zoubeidi A, Anderson S, Bell RH, et al: From sequence to molecular pathology, and a mechanism driving the neuroendocrine phenotype in prostate cancer. J Pathol 227: 286-297, 2012

30. Sharma NL, Massie CE, Ramos-Montoya A, Zecchini V, Scott HE, Lamb AD, MacArthur S, Stark R, Warren AY, Mills IG and Neal DE: The androgen receptor induces a distinct transcriptional program in castration-resistant prostate cancer in man. Cancer Cell 23: 35-47, 2013.

31. Kim J, Jin H, Zhao JC, Yang YA, Li Y, Yang X, Dong X and Yu J: FOXA1 inhibits prostate cancer neuroendocrine differentiation. Oncogene 36: 4072-4080, 2017. 
32. Ooi L and Wood IC: Chromatin crosstalk in development and disease: Lessons from REST. Nat Rev Genet 8: 544-554, 2007.

33. Akamatsu S, Wyatt AW, Lin D, Lysakowski S, Zhang F, Kim S, Tse C, Wang K, Mo F, Haegert A, et al: The placental gene PEG10 promotes progression of neuroendocrine prostate cancer. Cell Rep 12: 922-936, 2015.

34. Cully M: Anticancer drugs: Cutting down on prostate cancer metastases. Nat Rev Drug Discov 18: 17, 2018.

35. Thiery JP, Acloque H, Huang RY and Nieto MA: Epithelial-mesenchymal transitions in development and disease. Cell 139: 871-890, 2009.

36. O'Brien SJ, Carter JV, Burton JF, Oxford BG, Schmidt MN Hallion JC and Galandiuk S: The role of the miR-200 family in epithelial-mesenchymal transition in colorectal cancer: A systematic review. Int J Cancer 142: 2501-2511, 2018.

37. Hui Z, Zhanwei W, Xi Y, Jin L, Jing Z and Shuwen H: Construction of ceRNA coexpression network and screening of molecular targets in colorectal cancer. Dis Markers 2020: 2860582, 2020

38. Yang JD and Roberts LR: Epidemiology and management of hepatocellular carcinoma. Infect Dis Clin North Am 24: 899-919, viii, 2010.

39. Guo LM, Pu Y, Han Z, Liu T, Li YX, Liu M, Li X and Tang H: MicroRNA-9 inhibits ovarian cancer cell growth through regulation of NF-kappaB1. FEBS J 276: 5537-5546, 2009.

40. Wan HY, Guo LM, Liu T, Liu M, Li X and Tang H: Regulation of the transcription factor NF-kappaB1 by microRNA-9 in human gastric adenocarcinoma. Mol Cancer 9: 16, 2010.
41. Song Y, Li J, Zhu Y, Dai Y, Zeng T, Liu L, Li J, Wang H, Qin Y, Zeng $\mathrm{M}$, et al: MicroRNA-9 promotes tumor metastasis via repressing E-cadherin in esophageal squamous cell carcinoma. Oncotarget 5: 11669-11680, 2014.

42. Liu W, Gao G, Hu X, Wang Y, Schwarz JK, Chen JJ, Grigsby PW and Wang X: Activation of miR-9 by human papillomavirus in cervical cancer. Oncotarget 5: 11620-11630, 2014.

43. Medema JP: Cancer stem cells: The challenges ahead. Nat Cell Biol 15: 338-344, 2013.

44. Xie Y, Dang W, Zhang S, Yue W, Yang L, Zhai X, Yan Q and $\mathrm{Lu} \mathrm{J}$ : The role of exosomal noncoding RNAs in cancer. Mol Cancer 18: 37, 2019.

45. Hu J, Chen Y, Li X, Miao H, Li R, Chen D and Wen Z: THUMPD3-AS1 is correlated with non-small cell lung cancer and regulates self-renewal through miR-543 And ONECUT2. Onco Targets Ther 12: 9849-9860, 2019.

46. Grossfeld GD, Litwin MS, Wolf JS, Jr, Hricak H, Shuler CL, Agerter DC and Carroll PR: Evaluation of asymptomatic microscopic hematuria in adults: The American Urological Association best practice policy-part II: Patient evaluation, cytology, voided markers, imaging, cystoscopy, nephrology evaluation, and follow-up. Urology 57: 604-610, 2001.

47. Babjuk M, Burger M, Comperat EM, Gontero P, Mostafid AH, Palou J, van Rhijn BWG, Roupret M, Shariat SF, Sylvester R, et al: European association of urology guidelines on Non-muscle-invasive bladder cancer (TaT1 and Carcinoma in situ)-2019 update. Eur Urol 76: 639-657, 2019. 